\title{
Finite-Time Anti-Disturbance Inverse Optimal Attitude Tracking Control of Flexible Spacecraft
}

\author{
Chutiphon Pukdeboon and Anuchit Jitpattanakul \\ Department of Mathematics, Faculty of Applied Science, King Mongkut's University of Technology North Bangkok, \\ Bangkok 10800, Thailand \\ Correspondence should be addressed to Chutiphon Pukdeboon; cpd@kmutnb.ac.th
}

Received 4 September 2013; Accepted 25 November 2013

Academic Editor: Baoyong Zhang

Copyright (C) 2013 C. Pukdeboon and A. Jitpattanakul. This is an open access article distributed under the Creative Commons Attribution License, which permits unrestricted use, distribution, and reproduction in any medium, provided the original work is properly cited.

\begin{abstract}
We propose a new robust optimal control strategy for flexible spacecraft attitude tracking maneuvers in the presence of external disturbances. An inverse optimal control law is designed based on a Sontag-type formula and a control Lyapunov function. An adapted extended state observer is used to compensate for the total disturbances. The proposed controller can be expressed as the sum of an inverse optimal control and an adapted extended state observer. It is shown that the developed controller can minimize a cost functional and ensure the finite-time stability of a closed-loop system without solving the associated Hamilton-Jacobi-Bellman equation directly. For an adapted extended state observer, the finite-time convergence of estimation error dynamics is proven using a strict Lyapunov function. An example of multiaxial attitude tracking maneuvers is presented and simulation results are included to show the performance of the developed controller.
\end{abstract}

\section{Introduction}

Optimal control for spacecraft rotational problems has attracted a great deal of interest. The problem of optimal attitude control has been studied by many researchers (see, e.g., [1-4]). The main objective of optimal attitude control is to design a controller that stabilizes the attitude of a spacecraft system to an equilibrium state and minimizes some performance criterion for the stabilization process. Generally for linear systems, the linear quadratic regulator (LQR) is able to ensure an optimal and asymptotically stable solution. In [5] the LQR method was extended to a nonlinear control problem but more constraints were required to meet the optimality and stability conditions. Various nonlinear optimal control methods have been proposed for solving the attitude control problem. Sharma and Tewari [6] devised a Hamilton-Jacobi formulation for tracking attitude maneuvers of spacecraft to derive a nonlinear optimal control law. In [7] optimal controllers for a programmed motion of a rigid spacecraft were designed using the optimal Lyapunov approach. In $[8,9]$ state-dependent Riccati equation (SDRE) techniques were successfully applied to spacecraft attitude control. In [10] a class of globally asymptotically stabilizing controllers was developed for the complete attitude motion of a nonsymmetric rigid body. An inverse optimal control approach was presented in [11] to construct the optimal controller for regulation of the rigid body. Recently, attitude controller designs for rigid spacecraft using inverse optimal control schemes have been developed [12]. The inverse optimal control method incorporates the task of solving a Hamilton-Jacobi-Bellman equation and offers a globally asymptotically stabilizing control law which is optimal with respect to a performance index. Sontag's formula [13] uses the directional information supplied by a control Lyapunov function (CLF). Freeman and Kokotovic [14] have shown that every CLF solves the Hamilton-Jacobi-Bellman (HJB) equation associated with a meaningful cost. In other words, if we have a CLF for a nonlinear system, we can compute the resulting optimal control law without solving the HJB equation.

As extensions of the above studies, optimal control and robust control have been merged to obtain robust optimal control laws. Various methods for developing robust optimal controllers for the attitude control of a rigid spacecraft have 
been proposed in the literature. Kang [15] used nonlinear $H_{\infty}$ control to design a stabilizing feedback control for the spacecraft tracking problem. Luo et al. [16] developed an $H_{\infty}$ inverse optimal adaptive controller for attitude tracking of spacecraft. Adaptive control and nonlinear $H_{\infty}$ control have also been merged to design robust optimal controllers. Park [17] proposed a robust optimal control scheme for attitude stabilization and used a minimax approach and inverse optimal approach to examine the optimality property of this control law. Due to its lower dependence on model information and its strong capability for estimating disturbance and simple structure, an extended state observer (ESO) [18] has been widely used to deal with various kinds of engineering control problems such as flight control and chemical process control. In [19] the ESO based disturbance rejection control approach has been addressed for attitude tracking of a rigid spacecraft. The vibration effect of flexible appendages was not considered in the attitude control design. An alternative way to design a robust optimal controller is to use an optimal sliding mode controller design scheme. Sliding mode control (SMC) is a very effective approach when applied to a system with disturbances which satisfy the matched uncertainty condition [20]. Pukdeboon and Zinober $[21,22]$ have developed robust optimal control laws based on the optimal sliding mode control technique for attitude tracking of spacecraft. However, since the attitude control system of flexible spacecraft is quite complicated, optimal sliding mode control has rarely been studied for attitude control design. SDRE-based optimal sliding mode (SM) control and optimal Lyapunov-based SM control approaches have been used in [23] to design optimal controllers for attitude stabilization of flexible spacecraft. However, the optimal controllers developed in that paper contain some drawbacks. The SDRE approach usually provides only local asymptotic stability while for the optimal Lyapunov approach it is a formidable task to choose a Lyapunov function to satisfy the partial differential equation derived from the Krasovskii theorem [24].

In this paper, a novel control methodology for flexible spacecraft attitude maneuvers is proposed in the presence of external disturbances. First, an inverse optimal controller for stabilizing systems is designed based on a Sontag-type formula $[25,26]$ and a finite-time control Lyapunov function (FTCLF) $[27,28]$. Then, the total disturbance is estimated by an adapted $\mathrm{ESO}$ which is a modified version of the traditional ESO in $[18,29]$. The stability of the traditional ESO [18] has been proved using the self-stable region (SSR) approach [30], but this approach takes many steps and is rather complicated. In this paper the finite-time convergence of an adapted ESO is proved by using a strict Lyapunov function. The proposed new attitude controller for flexible spacecraft enforces tracking motion, robustness, and optimality with respect to a family of cost functionals and achieves disturbance rejection.

The main contributions of this paper are as follows.

(i) An inverse optimal control method for flexible spacecraft attitude tracking maneuvers is proposed for the first time in this paper. (ii) A second-order sliding mode based disturbance compensator is developed and combined with the proposed attitude controller. The necessity of a compensator is also discussed.

(iii) The hybrid control method is used to develop a controller with complete robustness under the system uncertainty and external disturbances.

This paper is organized as follows. Section 2 introduces some preliminary results which are required for the following discussion. In Section 3 the dynamic equations of a flexible spacecraft and the attitude kinematics $[31,32]$ are described. Section 4 provides the problem statement and control objective. Section 5 proposes a finite-time inverse optimal control design with the FTCLF concepts. We design an inverse optimal controller that provides the convergence of system states to the desired attitude motion. In Section 6, an adapted ESO method is used to develop an anti-disturbance feedback controller. The finite-time convergence of estimation error dynamics is proved using a strict Lyapunov function. In Section 7, an example of spacecraft attitude maneuvers is presented to illustrate the performance of the developed control law. In Section 8, we present conclusions.

\section{Mathematical Preliminaries}

2.1. Finite-Time Stability. We now restate the concepts related to finite-time stability [33, 34].

Definition 1 (see [33]). Consider a time invariant system in the form of

$$
\dot{x}=f(x), \quad f(0)=0 \quad x \in \mathscr{R}^{n},
$$

where $f: \widehat{U}_{0} \rightarrow \mathscr{R}^{n}$ is continuous on an open neighborhood $\widehat{U}_{0}$ of the origin. The equilibrium $x=0$ of the system is (locally) finite-time stable if (i) it is asymptotically stable, in $\widehat{U}$, an open neighborhood of the origin, with $\widehat{U} \subset \widehat{U}_{0}$ and (ii) it is finite-time convergent in $\widehat{U}$; that is, for any initial condition $x_{0} \in \widehat{U} \backslash\{0\}$, there is a settling time $T>0$ such that every solution $x\left(t, x_{0}\right)$ of system (1) is defined with $x\left(t, x_{0}\right) \in \widehat{U} \backslash\{0\}$ for $t \in[0, T]$ and satisfies

$$
\lim _{t \rightarrow T\left(x_{0}\right)} x\left(t, x_{0}\right)=0
$$

and $x\left(t, x_{0}\right)=0$, if $t \geq T$. Moreover, if $\widehat{U}=\mathscr{R}^{n}$, the origin is globally finite-time stable.

Definition 2. Consider a controlled system

$$
\dot{x}=f(x)+b(x) u, \quad x \in \mathscr{R}^{n}, u \in \mathscr{R}^{m},
$$

with $b(x) \neq 0$. It is finite-time stabilizable if there is a feedback law $u(x)$ such that $x=0$ is a (locally) finite-time stable equilibrium of the closed-loop system.

Lemma 3 (see [34]). Consider the nonlinear system described in (1). Suppose there is a $C^{1}$ function $V(x)$ defined on a neighborhood $\widehat{U} \subset U_{0} \subset \mathscr{R}^{n}$ of the origin such that the 
following conditions hold: (i) $V(x)$ is positive definite on $\widehat{U}$ and (ii) there are real numbers $\varrho>0$ and $0<\iota<1$, such that

$$
\dot{V}(x)+\varrho(V(x))^{\iota} \leq 0, \quad x \in \widehat{U} \backslash\{0\} .
$$

Then, the origin of system (1) is locally finite-time stable, with its settling time

$$
T \leq \frac{V\left(x_{0}\right)^{1-\iota}}{\varrho(1-\iota)} .
$$

If $\widehat{U}=\mathscr{R}^{n}$ and $V(x)$ is radially unbounded, then the origin of system (1) is globally finite-time stable.

2.2. Finite-Time Control Lyapunov Function. Based on the definition of a finite-time control Lyapunov function (FTCLF) in $[27,28]$, we provide the following definitions.

Definition 4 (see $[27,28]$ ). Let $U_{0}$ be a neighborhood of the origin. A positive-definite and $C^{1}$ function $V: U_{0} \rightarrow R^{+}$ is called a finite-time control Lyapunov function (FTCLF) of system (3) if there exist real numbers $c_{1}>0$ and $\alpha \in(0,1)$ such that for all $x \in U_{0} \backslash\{0\}$,

$$
\inf _{u}\left\{L_{f} V(x)+L_{b} V(x) u\right\} \leq-c_{1}(V(x))^{\alpha},
$$

where $L_{f} V(x)$ and $L_{b} V(x)$ are the Lie derivatives of $V$ along the solution of system (3).

Note that the Lie derivative of $V$ with respect to $h: R^{n} \rightarrow$ $R^{n}$ is defined as the inner product of $h$ and the gradient of $V$; that is, $L_{h} V(x)=(\partial V / \partial x) h$.

Obviously, if (6) holds, then for all $\in U_{0} \backslash\{0\}$, the condition that $V(x)$ is a FTCLF of (3) is precisely the statement

$$
L_{b} V=0 \Longrightarrow L_{f} V<-c_{1}(V(x))^{\alpha} .
$$

In fact, if there exists a positive-definite and $C^{1}$ function $V: U_{0} \rightarrow R^{+}$such that (7) holds, then $V$ is a FTCLF of system (3). Note that if $V(x)$ is a continuous positivedefinite function, then $L_{f} V(x)$ and $L_{b} V(x)$ can be seen as Dini derivatives.

Definition 5 (see [27]). $V(x)$ is said to satisfy the extended small control property with respect to system (3) if, for all $\epsilon>$ 0 , there exists $\varepsilon>0$ such that if $x \neq 0$ and $\|x\|<\varepsilon$, then there exists a certain $u$ with $\|u\|<\epsilon$, such that $L_{f} V(x)+L_{b} V(x) u \leq$ $-c_{1}(V(x))^{\alpha}$, where $c_{1}>0$ and $\alpha \in(0,1)$.

2.3. The Inverse Optimal Control Problem. This subsection considers the design of finite-time inverse optimal control. A feedback control law $u(x)$ for the system (3) will be designed such that the closed-loop system is finite-time stable at the equilibrium $x=0$ and minimizes the cost functional

$$
I\left(u, x, x_{0}\right)=\int_{0}^{T(x)}\left(l(x)+u^{T} R u\right) d t
$$

where $l(x) \geq 0$ and $R(x)>0$ for all $x$ and $T(x)$ is the settling time function.
According to the theorem in $[11,27]$, if $V(x)$ is a FTCLF for the system (3), then a stabilizing control law

$$
u(x)=\alpha^{*}(x)=-\frac{1}{2} R^{-1}(x)\left(L_{b} V(x)\right)^{T}
$$

solves an inverse optimal control problem for the system (3) with respect to the cost functional (8).

Moreover, if we choose

$$
l(x)=-L_{f} V(x)-\frac{1}{2} L_{b} V(x) \alpha^{*}(x),
$$

then $l(x) \geq 0$ is achieved with the control law $u=(1 / 2) \alpha^{*}$. Next, substituting $u=(1 / 2) \alpha^{*}$ into (10), we obtain the HJB equation

$$
l(x)=-L_{f} V(x)+\frac{1}{4} L_{b} V(x) R^{-1}(x)\left(L_{b} V(x)\right)^{T} \geq 0 .
$$

Then, using the concept in [27], we have that $V(x)$ is the solution of the HJB equation (11).

Remark 6. In the inverse optimal approach, a finite-time stabilizing feedback control law $u(x)$ is designed first and then it is shown that the feedback law is to find $l(x) \geq 0$ and $R(x)>0$ such that $u$ optimizes (8). The problem is inverse because the functions $l(x)$ and $R(x)$ are a posteriori found by the stabilizing feedback law, rather than a priori selected by the designer.

\section{Mathematical Model of Flexible Spacecraft}

We now briefly explain the use of quaternions for description of the attitude error. We define the quaternion $Q=$ $\left[\begin{array}{ll}q^{T} & q_{4}\end{array}\right]^{T} \in \mathscr{R}^{3} \times \mathscr{R}$ with $q=\left[\begin{array}{lll}q_{1} & q_{2} & q_{3}\end{array}\right]^{T} \in \mathscr{R}^{3}$ and

$$
Q_{d}=\left[\begin{array}{ll}
q_{d}^{T} & q_{4 d}
\end{array}\right]^{T}
$$

where $q_{d}=\left[\begin{array}{lll}q_{1 d} & q_{2 d} & q_{3 d}\end{array}\right]^{T} \in \mathscr{R}^{3}$ is the desired reference attitude. The quaternion for the attitude error is $Q_{e}=$ $\left[\begin{array}{ll}q_{e}^{T} & q_{4 e}\end{array}\right]^{T} \in \mathscr{R}^{3} \times \mathscr{R}$ with $q_{e}=\left[\begin{array}{lll}q_{1 e} & q_{2 e} & q_{3 e}\end{array}\right]^{T} \in \mathscr{R}^{3}$. Using the multiplication law for quaternions, we then obtain

$$
Q_{e}=\left[\begin{array}{c}
q_{4 d} q-q_{4} q_{d}-q_{d}^{\times} q \\
q_{4} q_{4 d}+q^{T} q_{d}
\end{array}\right]
$$

subject to the constraint

$$
Q_{e}^{T} Q_{e}=\left(q^{T} q+q_{4}^{2}\right)\left(q_{d}^{T} q_{d}+q_{4 d}^{2}\right)=1
$$

In $(13) q_{d}^{\times}$is given by

$$
q_{d}^{\times}=\left[\begin{array}{ccc}
0 & -q_{3 d} & q_{2 d} \\
q_{3 d} & 0 & -q_{1 d} \\
-q_{2 d} & q_{1 d} & 0
\end{array}\right] .
$$


Remark 7. A quaternion consists of the scalar $q_{4}$ and the three-dimensional vector $q$, so it has four components. The scalar term is used for avoidance of singular points in the attitude representation [35]. The quaternion kinematics equation is required to be solved for all four components. However, to indicate the maneuver of the spacecraft, it is sufficient to use only the vector $q$ because this vector properly represents both Euler axis and Euler angle. Furthermore, the scalar $q_{4}$ can be calculated easily using the vector $q$ and the condition $\|Q\|=1$. For more details of quaternion and other attitude representation see [31, 35].

The kinematic equation for the attitude error can then be expressed as (see, $[31,36])$

$$
\dot{Q}_{e}=\frac{1}{2}\left[\begin{array}{c}
T\left(Q_{e}\right) \\
-q_{e}^{T}
\end{array}\right] \omega_{e},
$$

where $T\left(Q_{e}\right)=q_{e}^{\times}+q_{4 e} I_{3}$ with $I_{3}$ being the $3 \times 3$ identity matrix. as [32]

The equation governing a flexible spacecraft is expressed

$$
\begin{gathered}
J \dot{\omega}+\delta^{T} \ddot{\eta}=-\omega^{\times}\left(J \omega+\delta^{T} \dot{\eta}\right)+u+d, \\
\ddot{\eta}+C \dot{\eta}+K \eta=-\delta \dot{\omega},
\end{gathered}
$$

where $J=J^{T}$ is the total inertia matrix of the spacecraft, $\eta$ is the modal displacement, and $\delta$ is the coupling matrix between the central rigid body and the flexible attachments. $u \in \mathscr{R}^{3}$ denotes the control input, $d \in \mathscr{R}^{3}$ represents the external disturbances, and $K$ and $C$ denote the stiffness and damping matrices, respectively, which are defined as

$$
\begin{gathered}
K=\operatorname{diag}\left(\omega_{n i}^{2}, i=1,2, \ldots, N\right), \\
C=\operatorname{diag}\left(2 \zeta_{i} \omega_{n i}, i=1,2, \ldots, N\right)
\end{gathered}
$$

with damping $\zeta_{i}$ and natural frequency $\omega_{n i}$.

We denote by $\omega_{d}=\left[\begin{array}{lll}\omega_{1 d} & \omega_{2 d} & \omega_{3 d}\end{array}\right]^{T}$ the desired angular velocity and by $\omega_{e}=\omega-\omega_{d}$ as the angular velocity error. Let

$$
\vartheta=\left[\begin{array}{l}
\eta \\
\psi
\end{array}\right],
$$

where $\psi=\dot{\eta}+\delta \omega_{e}$. The relative dynamic equation can be written as [36]

$$
\begin{gathered}
\dot{\omega}_{e}=J_{m b}^{-1}\left[-\left(\omega_{e}^{\times}+\omega_{d}^{\times}\right)\left(J_{m b} \omega_{e}+\delta^{T} \psi+J \omega_{d}\right)\right. \\
\left.+\delta^{T}\left(C \psi+K \eta-C \delta \omega_{e}\right)+u+d\right]-\dot{\omega}_{d}, \\
\dot{\vartheta}=A \vartheta+B \omega_{e}+D \dot{\omega}_{d},
\end{gathered}
$$

where $\dot{\omega}_{d}$ is the first time derivative of $\omega_{d}$. The matrices $J_{m b}$, $A B$, and $D$ are given as

$$
\begin{gathered}
J_{m b}=J-\delta^{T} \delta \\
A=\left[\begin{array}{cc}
0_{4 \times 4} & I_{4} \\
-K & -C
\end{array}\right], \quad B=\left[\begin{array}{c}
-\delta \\
C \delta
\end{array}\right], \quad D=\left[\begin{array}{c}
0_{4 \times 3} \\
-\delta
\end{array}\right] .
\end{gathered}
$$

Clearly, $A$ is a Hurwitz matrix.
If we let

$$
x(t)=\left[\begin{array}{llll}
\omega_{e}^{T} & q_{4 e} & q_{e}^{T} & \vartheta^{T}
\end{array}\right]^{T},
$$

then the spacecraft systems (16), (17a), and (17b) can be expressed in the state space form as

$$
\begin{array}{cc}
\dot{x}=f(x)+b(x) u+b(x) d, & x(0)=x_{0}, \\
f(x)=\left[\begin{array}{c}
J_{m b}^{-1} \Xi\left(\omega_{e}, \omega_{d}, \dot{\omega}_{d}\right) \\
-0.5 q_{e}^{T} \omega_{e} \\
0.5\left(q_{e}^{\times}+q_{4 e} I_{3}\right) \omega_{e} \\
A \vartheta+B \omega_{e}+D \dot{\omega}_{d}
\end{array}\right], \quad b(x)=\left[\begin{array}{c}
J_{m b}^{-1} \\
0_{1 \times 3} \\
0_{3 \times 3} \\
0_{8 \times 3}
\end{array}\right],
\end{array}
$$

where

$$
\begin{aligned}
\Xi\left(\omega_{e}, \omega_{d}, \dot{\omega}_{d}\right)= & -\omega_{e}^{\times} J_{m b} \omega_{e}-\omega_{e}^{\times} \delta^{T} \psi-\omega_{e}^{\times} J \omega_{d}-\omega_{d}^{\times} J_{m b} \omega_{e} \\
& -\omega_{d}^{\times} \delta^{T} \psi-\omega_{d}^{\times} J \omega_{d}+\delta^{T}\left(C \psi+K \eta-C \delta \omega_{e}\right) \\
& -J_{m b} \dot{\omega}_{d} .
\end{aligned}
$$

\section{Problem Statement}

In this work we consider tracking maneuvers. The control objective is to realize desired rotations of flexible spacecraft in the presence of external disturbances and minimize a cost functional. In other words, we shall find a controller $u$ subject to (24) such that for all initial conditions the desired rotations are achieved

$$
\lim _{t \rightarrow T} q_{e}=0, \quad \lim _{t \rightarrow T} q_{4 e}=1, \quad \lim _{t \rightarrow T} \omega_{e}=0
$$

and the cost functional (8) is minimized. Note that, when $q_{e} \rightarrow 0$, we have $q_{4 e} \rightarrow 1$, due to the constraint relation $q_{e}^{T} q_{e}+q_{4 e}^{2}=1$.

\section{Inverse Optimal Controller Design for Flexible Spacecraft}

In this section, we first propose a finite-time inverse optimal controller for stabilizing the complete attitude motion of flexible spacecraft in the presence of external disturbances.

In order to design this controller for solving the finitetime inverse optimal problem we first choose a FTCLF for the system (24) as the following candidate positive-definite function:

$$
\begin{aligned}
V(x)= & \frac{1}{2} \omega_{e}^{T} J_{m b} \omega_{e}+\beta\left(q_{4 e}-1\right)^{2}+\beta q_{e}^{T} q_{e} \\
& +\frac{1}{2} \vartheta^{T} P \vartheta+\gamma q_{e}^{T} J_{m b} \omega_{e},
\end{aligned}
$$

where $\beta$ and $\gamma$ are nonnegative constants and $P$ is a positivedefinite matrix that is a solution of the Lyapunov equation $A^{T} P+P A=-Q_{A}$ with a positive-definite matrix $Q_{A}$. 
Assumption 8. The desired angular velocity vector and its first time derivative are bounded and satisfy the following conditions:

$$
\left\|\omega_{d}\right\| \leq W_{1}, \quad\left\|\dot{\omega}_{d}\right\| \leq W_{2},
$$

where $W_{1}$ and $W_{2}$ are positive constants.

We next show that the function $V(x)$ defined in (28) is a FTCLF for the system (24) by using the following lemma.

Lemma 9. In the absence of disturbance vector, under Assumption 8, the positive definite $V$ defined in (28) is a FTCLF for the spacecraft tracking system (24).

Proof. Since $J_{m b}$ is symmetric positive definite, we can write $V(x)$ as

$$
V=\bar{x}^{T} \Omega \bar{x}+\beta\left(q_{4 e}-1\right)^{2},
$$

where $\bar{x}(t)=\left[\begin{array}{lll}\omega_{e}^{T} & q_{e}^{T} & \vartheta^{T}\end{array}\right]^{T}$, and

$$
\Omega=\frac{1}{2}\left[\begin{array}{ccc}
J_{m b} & \gamma J_{m b} & 0_{3 \times 8} \\
\gamma J_{m b} & 2 \beta I_{3} & 0_{3 \times 8} \\
0_{8 \times 3} & 0_{8 \times 3} & P
\end{array}\right]
$$

The conditions for $V(x)$ to be positive definite are

$$
\beta>0, \quad 2 \beta J_{m b}>\gamma^{2} J_{m b}^{2} .
$$

Also, using (28) we can obtain the following inequalities:

$$
\begin{aligned}
& \sigma_{\min }(\Omega)\|\bar{x}\|^{2}+\beta\left(q_{4 e}-1\right)^{2} \\
& \quad \leq V \leq \sigma_{\max }(\Omega)\|\bar{x}\|^{2}+\beta\left(q_{4 e}-1\right)^{2},
\end{aligned}
$$

where $\|\bar{x}\|$ denotes the Euclidean norm of $\bar{x}$ and $\sigma_{\min }(\Omega)$ and $\sigma_{\max }(\Omega)$ denote the minimum and maximum singular values of the matrix $\Omega$.

The first time derivative of $V$ can be obtained as

$$
\begin{gathered}
\frac{\partial V(x)}{\partial x}=\left[\begin{array}{c}
J_{m b} \omega_{e}+\gamma J_{m b} q_{e} \\
2 \beta\left(q_{4 e}-1\right) \\
2 \beta J_{m b} \omega_{e}+\gamma q_{e} \\
\vartheta^{T} P
\end{array}\right], \\
{\left[\frac{\partial V(x)}{\partial x}\right]^{T}} \\
=\left[\omega_{e}^{T} J_{m b}+\gamma q_{e}^{T} J_{m b}\right. \\
\left.2 \beta\left(q_{4 e}-1\right) \omega_{e}^{T} J_{m b} \beta+q_{e}^{T} \gamma \vartheta^{T} P\right] .
\end{gathered}
$$

Thus, we have

$$
\begin{aligned}
L_{b} V & \\
= & {\left[\omega_{e}^{T} J_{m b}+\gamma q_{e}^{T} J_{m b} 2 \beta\left(q_{4 e}-1\right) \omega_{e}^{T} \beta J_{m b}+q_{e}^{T} \gamma \vartheta^{T} P\right] } \\
& \times\left[\begin{array}{c}
J_{m b}^{-1} \\
0_{1 \times 3} \\
0_{3 \times 3} \\
0_{8 \times 3}
\end{array}\right] \\
= & \omega_{e}^{T}+q_{e}^{T} \gamma .
\end{aligned}
$$

Therefore, if $L_{b} V=0$, we have

$$
\omega_{e}^{T}=-\gamma q_{e}^{T} .
$$

Next, we show that for all $x \neq 0$, if $L_{b} V=0$, then $L_{f} V<$ $-c_{2} V^{1 / 2}$ with $c_{2}$ being a positive constant. Here, $L_{f} V$ can be expressed as

$$
\begin{aligned}
L_{f} V= & \left(\omega_{e}^{T} J_{m b}+\gamma q_{e}^{T} J_{m b}\right)\left(J_{m b}^{-1} \Xi\left(\omega_{e}, \omega_{d}, \dot{\omega}_{d}\right)\right) \\
& +2 \beta\left(q_{4 e}-1\right)\left(-\frac{1}{2} q_{e}^{T} \omega_{e}\right)+\beta q_{e}\left(q_{e}^{\times}+q_{4 e} I_{3}\right) \omega_{e} \\
& +\vartheta^{T} P\left(A \vartheta+B \omega_{e}+D \dot{\omega}_{d}\right) .
\end{aligned}
$$

Substituting (37) in (38), we obtain

$$
\begin{aligned}
L_{f} V= & \beta\left(q_{4 e}-1\right) \gamma q_{e}^{T} q_{e}+\beta q_{e}\left(q_{e}^{\times}+q_{4 e} I_{3}\right)\left(-\gamma q_{e}\right) \\
& +\vartheta^{T} P A \vartheta+\vartheta^{T} P B(-\gamma q)+\vartheta^{T} P D \dot{\omega}_{d} .
\end{aligned}
$$

Since $q_{e}^{\times} q_{e}=0$, (39) becomes

$$
\begin{aligned}
L_{f} V & \leq-\beta \gamma q_{e}^{T} q_{e}-\frac{1}{2} \vartheta^{T} Q_{A} \vartheta-\gamma \vartheta^{T} P B q_{e}+\|\vartheta\|\|P D\| W_{2} \\
& \leq-\omega^{T} \Pi_{1} \omega+\|\vartheta\|\|P D\| W_{2}
\end{aligned}
$$

where

$$
\omega=\left[\begin{array}{c}
q_{e} \\
\vartheta
\end{array}\right], \quad \Pi_{1}=\left[\begin{array}{cc}
\beta \gamma & \frac{\gamma}{2} P B \\
\frac{\gamma}{2} P B & \frac{1}{2} Q_{A}
\end{array}\right] .
$$

With proper parameters $\beta$ and $\gamma$, one can obtain that $\Pi_{1}$ is positive definite

$$
\begin{aligned}
L_{f} V & \leq-\sigma_{\min }\left(\Pi_{1}\right)\|\omega\|^{2}+\|\omega\|\|P D\| W_{2} \\
& \leq-\left(\sigma_{\min }\left(\Pi_{1}\right)\|\omega\|-\|P D\| W_{2}\right)\|\omega\| .
\end{aligned}
$$

From (33) we have $\sigma_{\min }(\Omega)\|\bar{x}\|^{2} \leq V$. Using $\|\omega\| \leq\|\bar{x}\|$, one has $\sigma_{\min }(\Omega)\|\omega\|^{2} \leq V$ and it follows that $\|\omega\| \leq$ $V^{1 / 2} / \sqrt{\sigma_{\min }(\Omega)}$. Thus, (42) becomes

$$
L_{f} V \leq-\left(\sigma_{\min }\left(\Pi_{1}\right)\|\omega\|-\|P D\| W_{2}\right) \frac{V^{1 / 2}}{\sqrt{\sigma_{\min }(\Omega)}} \leq-c_{2} V^{1 / 2},
$$

where $c_{2}=\left(\sigma_{\min }\left(\Pi_{1}\right)\|\omega\|-\|P D\| W_{2}\right) / \sqrt{\sigma_{\min }(\Omega)}$.

We know that there exist proper parameters $\beta$ and $\gamma$ such that $\Pi_{1}$ is positive definite and $\|\omega\|>\|P D\| W_{2} / \sigma_{\min }\left(\Pi_{1}\right)$ can be achieved with these parameters. So, if $L_{b} V=0$, then for all $x \neq 0$ we can obtain $L_{f} V \leq-c_{2} V^{1 / 2}$. This guarantees that the candidate $V(x)$ is a FTCLF for system (24).

Next, the main results of our proposed anti-disturbance inverse optimal control for the spacecraft model are presented.

Theorem 10. Let Assumption 8 hold. The following dynamic feedback control law

$$
u=u^{*}-\widehat{d}
$$

with 


$$
u^{*}= \begin{cases}-\varphi^{T}(x) \frac{\psi(x)+c_{3}(V(x))^{1 / 2}+\sqrt{\left(\psi(x)+c_{3}(V(x))^{1 / 2}\right)^{2}+\|\varphi(x)\|^{4}}}{\|\varphi(x)\|^{2}}, & \text { if }\|\varphi(x)\| \neq 0 \\ 0, & \text { if }\|\varphi(x)\|=0,\end{cases}
$$

where $c_{3}$ is a positive constant, $\hat{d}$ is an estimated value of $d$ which will be defined later, $\psi(x)=L_{f} V$, and $\varphi(x)=L_{b} V$, finite-time stabilizes the spacecraft system (24).

Proof. We show that the control law $u$ in (44) is a stabilizing controller for attitude control system in (24). Consider the smooth positive-definite radially unbounded function $V(x)$ in (28) as the Lyapunov function. The time derivative of the $V(x)$ is

$$
\begin{aligned}
\dot{V}= & \frac{\partial V}{\partial x}(f(x)+b(x) u+b(x) d) \\
= & L_{f} V+L_{b} V\left(u^{*}-\widehat{d}\right)+\varphi(x) d \\
= & \psi(x)-\varphi^{T}(x) \varphi(x) \\
& \quad \times \frac{\psi(x)+c_{3}(V(x))^{1 / 2}+\sqrt{\left(\psi(x)+c_{3}(V(x))^{1 / 2}\right)^{2}+\|\varphi(x)\|^{4}}}{\|\varphi(x)\|^{2}}
\end{aligned}
$$

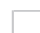

$$
\begin{aligned}
= & -\sqrt{\left(\psi(x)-c_{3}(V(x))^{1 / 2}\right)^{2}+\|\varphi(x)\|^{4}} \\
& -c_{3}(V(x))^{1 / 2}-\varphi^{T}(x)(\widehat{d}-d) \\
\leq & -c_{3}(V(x))^{1 / 2}-\varphi^{T}(x)(\widehat{d}-d) .
\end{aligned}
$$

It has been shown that if $\widehat{d}$ converges to $d$, then $e_{2}=$ $\widehat{d}-d$ converges to zero in finite time. This means that an appropriate $c_{3}$ can be selected such that $\dot{V} \leq-c_{3}(V(x))^{1 / 2}$ is achieved. By Lemma 3, the finite-time stability of closed-loop system is ensured. This completes the proof.

Next, we show that if the disturbance estimate $\widehat{d}$ in (44) and disturbance term $d$ in (24) are ignored, then the feedback stabilizing controller $u$ in (44) solves the inverse optimal control problem.

Theorem 11. Consider the system (24) for which Assumption 8 holds. Then the following dynamic feedback control law

$$
\begin{aligned}
u^{*} & =-R\left(e, e_{0}, \omega_{e}\right)^{-1} \varphi(x) \\
& = \begin{cases}\psi(x)+c_{3}(V(x))^{1 / 2}+\sqrt{\left(\psi(x)+c_{3}(V(x))^{1 / 2}\right)^{2}+\|\varphi(x)\|^{4}} & \text { if }\|\varphi(x)\| \neq 0 \\
-\varphi^{T}(x) \frac{\psi(x) \|^{2}}{0,} & \text { if }\|\varphi(x)\|=0\end{cases}
\end{aligned}
$$

solves the inverse optimal assignment problem for the attitude tracking system (24) by minimizing the cost functional (8).

Proof. With $\tilde{d}=0$, using the control law $u$ one obtains $\dot{V} \leq-c_{3}(V(x))^{1 / 2}$. Letting $u_{1}=(1 / 2) u^{*}$ we also obtain $\dot{V} \leq-c_{3}(V(x))^{1 / 2} \leq 0$. Next, choosing

$$
l(x)=-\psi(x)-\varphi(x) u_{1}
$$

it can be ensured that $l(x) \geq 0$. This shows that $l(x)$ is positive semidefinite in $q_{e}, q_{4 e}$, and $\omega_{e}$. Therefore this $l(x)$ is a meaningful cost function for the attitude control problem, penalizing on $q_{e}, q_{4 e}$, and $\omega_{e}$, as well as the control effort $u$. Substituting $l(x)$ into the cost functional (8), we obtain the optimal cost

$$
I\left(u^{*}, x, x_{0}\right)=V\left(x_{0}\right)
$$

for every $x_{0} \in D$.
Remark 12. It should be noted that most existing inverse optimal attitude control approaches have been developed for attitude motions of a rigid spacecraft $[16,17]$. For a flexible spacecraft the vibration of flexible appendages induced by an orbiting attitude slewing operation may degrade the attitude pointing accuracy. In this paper, we develop an inverse optimal attitude maneuver controller for a flexible spacecraft system which is significantly different from the controllers in $[16,17]$, where the effects of vibration of flexible appendages were not considered.

\section{Anti-Disturbance Inverse Optimal Control with Extended State Observer}

Due to the great advances in nonlinear control theory, the observer-based controller is now one of the most common schemes in industrial applications. The extended state observer (ESO) mentioned in $[18,28]$ has high efficiency 
in accomplishing nonlinear dynamic estimation. We know that use of the ESO dynamics of the observer error gives convergence into a residual set of zero. The convergence proof has been shown using the SSR approach [30]. However, this method takes many steps and is quite complicated. In this paper an adapted ESO which is a modified version of the traditional ESO is presented and the finite-time stability of the adapted ESO system is investigated using the strict Lyapunov function.

We now consider the coordinate transformation of the spacecraft model into the following form:

$$
z=\omega_{e}+\lambda q_{e}
$$

where $\lambda$ is a positive constant. The time derivative of $z$ is

$$
\dot{z}=\dot{\omega}_{e}+\lambda \dot{q}_{e} .
$$

Substituting (14) and (17a) into (51), we obtain the auxiliary dynamics

$$
\dot{z}=J_{m b}^{-1} \Xi\left(\omega_{e}, \omega_{e}, \dot{\omega}_{d}\right)+J_{m b}^{-1} u+J_{m b}^{-1} d+\frac{\lambda}{2}\left(q_{e}^{\times}+q_{4 e} I_{3}\right) \omega_{e},
$$

which can be rewritten as

$$
\dot{z}=F+\bar{B} u+\bar{d}
$$

where

$$
\begin{gathered}
F=J_{m b}^{-1} \Xi\left(\omega_{e}, \omega_{d}, \dot{\omega}_{d}\right)+\frac{\lambda}{2}\left(q_{e}^{\times}+q_{4 e} I_{3}\right) \omega_{e}, \\
\bar{B}=J_{m b}^{-1}, \quad \bar{d}=J_{m b}^{-1} d .
\end{gathered}
$$

Here, the new disturbance variable $\bar{d}$ is introduced. Although we need to estimate $d$ in (24), it is simpler to first estimate $\bar{d}$ and then use the results to estimate $d$. Thus, we now consider the estimate for $\bar{d}$ in the ESO design.

We next consider the auxiliary system (53) with the adapted ESO technique. The ESO views the system model uncertainties and external disturbances as an added state to be estimated. Using this idea, a nonlinear ESO can be designed for estimating the disturbances $\bar{d}(t)$. We add an extended state $\chi$ to the state equations to represent the total disturbances $\bar{d}$. The system (53) then becomes

$$
\begin{gathered}
\dot{z}=F+\bar{B} u+\chi, \\
\dot{\chi}=g(t),
\end{gathered}
$$

where the function $g(t)$ is the estimated derivative of the disturbances $\bar{d}(t)$.

Assumption 13. The $i$ th component of $g(t)$ is bounded; that is, $g_{i}(t) \leq \bar{g}_{1}, i=1,2,3$.

Then the adapted ESO for the system (53) is proposed to be as follows:

$$
\begin{gathered}
E_{1}=Z_{1}-\eta, \\
\dot{Z}_{1}=Z_{2}+F+\bar{B} u-\lambda_{2} E_{1}, \\
\dot{Z}_{2}=-\lambda_{1} E_{1}-\mu \operatorname{sign}^{r}\left(E_{1}\right),
\end{gathered}
$$

where $E_{1}$ is the estimation error of the ESO, $Z_{1}$ and $Z_{2}$ are the observer output, $\lambda_{1}=\operatorname{diag}\left(\lambda_{11}, \lambda_{12}, \lambda_{13}\right)$, and $\lambda_{2}=$ $\operatorname{diag}\left(\lambda_{21}, \lambda_{22}, \lambda_{23}\right)$ with $\lambda_{1 i}>0$ and $\lambda_{2 i}>0$ being the observer gains. Here, the function $\operatorname{sign}^{r}\left(E_{1}\right)$ is defined as

$$
\begin{aligned}
& \operatorname{sign}^{r}\left(E_{1}\right) \\
& \quad=\left[\begin{array}{llll}
\left|E_{11}\right|^{r} \operatorname{sign}\left(E_{11}\right) & \left|E_{12}\right|^{r} \operatorname{sign}\left(E_{12}\right) & \left|E_{13}\right|^{r} \operatorname{sign}\left(E_{13}\right)
\end{array}\right]^{T}
\end{aligned}
$$

with $r \in(0,1)$.

Theorem 14. Let Assumption 13 hold. Consider the system (55) with the adaptive ESO (56). Then there exist positive observer gains $\lambda_{1 i}, \lambda_{2 i}$, and $\mu_{i}(i=1,2,3)$ and $r \in(0,1)$ such that the estimated states $Z_{1}$ and $Z_{2}$ finite-time converge into a residual set of actual states $z$ and $\bar{d}$, respectively.

Proof. To investigate the stability of the ESO system, one must consider an expression for the observer dynamics. We first define the observer errors $E_{1}=Z_{1}-\eta$ and $E_{2}=Z_{2}-x_{2}=$ $Z_{2}-\bar{d}$. The observer error dynamics are also expressed as

$$
\begin{gathered}
\dot{E}_{1}=E_{2}-\lambda_{2} E_{1}, \\
\dot{E}_{2}=-g(t)-\lambda_{1} E_{1}-\mu \operatorname{sign}^{r}\left(E_{1}\right) .
\end{gathered}
$$

Letting $E_{1}=e_{1}$ and $E_{2}=e_{2}+\lambda_{2} e_{1}$ the observer error dynamics (58) can be transformed to the scalar form $(i=$ $1,2,3)$ as

$$
\begin{gathered}
\dot{e}_{1 i}=e_{2 i}, \\
\dot{e}_{2 i}=-g_{i}(t)-\lambda_{1 i} e_{1 i}-\lambda_{2 i} e_{2 i}-\mu_{i}\left|e_{1 i}\right|^{r} \operatorname{sign}\left(e_{1 i}\right) .
\end{gathered}
$$

We define $v=\left[\left|e_{1 i}\right|^{(r+1) / 2} \operatorname{sign}\left(e_{1 i}\right) \quad e_{1 i} e_{2 i}\right]^{T}$. To prove the stability, we select the Lyapunov function

$$
\bar{V}=\frac{1}{2} v^{T} \Lambda \nu
$$

where

$$
\Lambda=\left[\begin{array}{ccc}
\frac{\mu_{i}}{r+1} & 0 & 0 \\
0 & \lambda_{1 i} & \frac{\lambda_{2 i}}{2} \\
0 & \frac{\lambda_{2 i}}{2} & 1
\end{array}\right]
$$

The matrix $\Lambda$ is positive definite if $\lambda_{2 i}$ and $\lambda_{1 i}$ are chosen to satisfy the condition

$$
\lambda_{1 i}>\frac{1}{4} \lambda_{2 i}^{2}
$$

Taking the time derivative of $\bar{V}$ and using (60), we obtain

$$
\dot{\bar{V}}=\left[\begin{array}{ll}
e_{1 i} & e_{2 i}
\end{array}\right]\left[\begin{array}{cc}
\lambda_{1 i} & \frac{\lambda_{2 i}}{2} \\
\frac{\lambda_{2 i}}{2} & 1
\end{array}\right]\left[\begin{array}{c}
\dot{e}_{1 i} \\
\dot{e}_{2 i}
\end{array}\right]+\mu_{i}\left|e_{1 i}\right|^{r} \operatorname{sign}\left(e_{1}\right) \dot{e}_{1 i}
$$


which can be further written as

$$
\begin{aligned}
\dot{\bar{V}}= & {\left[\begin{array}{ll}
e_{1 i} & e_{2 i}
\end{array}\right]\left[\begin{array}{cc}
\lambda_{1 i} & 0.5 \lambda_{2 i} \\
0.5 \lambda_{2 i} & 1
\end{array}\right] } \\
& \times\left[\begin{array}{l}
e_{2 i}(t)-\lambda_{1 i} e_{1 i}-\lambda_{2 i} e_{2 i}-\mu_{i}\left|e_{1 i}\right|^{r} \operatorname{sign}\left(e_{1}\right)
\end{array}\right] \\
& +\mu_{i}\left|e_{1 i}\right|^{r} \operatorname{sign}\left(e_{1}\right) \dot{e}_{1 i} .
\end{aligned}
$$

After some manipulation, the derivative of $V$ can be written as follows:

$$
\dot{\bar{V}}=-\frac{\lambda_{2 i}}{2} v^{T} \Pi_{2} v+\varrho v,
$$

where

$$
\Pi_{2}=\left[\begin{array}{ccc}
\mu_{i} & 0 & 0 \\
0 & \lambda_{1 i} & \frac{\lambda_{2 i}}{2} \\
0 & \frac{\lambda_{2 i}}{2} & 1
\end{array}\right], \quad \varrho=\left[\begin{array}{lll}
0 & -\frac{\lambda_{2 i}}{2} g_{i}(t) & -g_{i}(t)
\end{array}\right] .
$$

Letting $L=\left[\begin{array}{lll}0 & \left(\lambda_{2 i} / 2\right) \bar{g}_{1} & \bar{g}_{1}\end{array}\right]$, one obtains

$$
\begin{aligned}
\dot{\bar{V}} & \leq-\frac{\lambda_{2 i}}{2} \sigma_{\min }\left(\Pi_{2}\right)\|\nu\|^{2}+\|L\|\|\vartheta\| \\
& =-\left(0.5 \lambda_{2 i} \sigma_{\min }\left(\Pi_{2}\right)\|\nu\|-\|L\|\right)\|\nu\| .
\end{aligned}
$$

Using (60) we know that $\bar{V} / \sigma_{\max }(\Lambda) \leq\|\nu\|^{2} \leq \bar{V} / \sigma_{\min }(\Lambda)$. We obtain

$$
\dot{\bar{V}} \leq-\left(\sigma_{\min }\left(\Pi_{2}\right)\|\nu\|-\|L\|\right) \frac{\bar{V}^{1 / 2}}{\sqrt{\sigma_{\max }(\Lambda)}} .
$$

If $\sigma_{\min }\left(\Pi_{2}\right)\|\nu\|>\|L\|$, the error system (59) will finite-time converge to the region

$$
\|\nu\| \leq\left(\frac{\|L\|}{\sigma_{\min }(\Lambda)}\right) .
$$

It is obvious that the estimation errors are determined by the parameters $\lambda_{2 i}, \lambda_{1 i}$, and $\mu_{i}$. Basically, these parameters can be chosen such that (62) is satisfied. This makes the error states converge to region (69) in finite time.

Remark 15. It should be noticed that conditions for the stability of the adapted ESO (56) have been obtained in terms of positive gains $\lambda_{2 i}, \lambda_{1 i}$, and $\mu_{i}$ and $r \in(0,1)$ in (59) for the estimation errors. When suitable gains are chosen, $Z_{2}$ will be a precise estimate of $\bar{d}$ and the estimation error $E_{2}$ will converge to region (69) in finite time.

Using the results from the ESO system, the estimated disturbance $\widehat{d}$ is determined by $\widehat{d}=\bar{B}^{-1} Z_{2}$. Thus, the proposed anti-disturbance inverse optimal control can be obtained as

$$
u=u^{*}-\bar{B}^{-1} Z_{2} \text {. }
$$

With suitable control gains defined by the inverse optimal control approach based on FTCLF concept, the optimal feedback controller (70) contains both optimality and robustness performance to attenuate external disturbances.

\section{Simulation Results}

An example of attitude control of flexible spacecraft is presented with numerical simulations. The performance of our proposed controller (70) is compared with the performances of the optimal Lyapunov sliding mode (SM) controller (4.3) in [23] and robust finite-time controller (23) in [37]. The spacecraft is assumed to have the nominal inertia matrix [38]

$$
J=\left[\begin{array}{ccc}
350 & 3 & 4 \\
3 & 270 & 10 \\
4 & 10 & 190
\end{array}\right] \mathrm{kg} \cdot \mathrm{m}^{2}
$$

and coupling matrices

$$
\delta=\left[\begin{array}{ccc}
6.45637 & 1.27814 & 2.15629 \\
-1.25619 & 0.91756 & -1.67264 \\
1.11678 & 2.48901 & -0.83674 \\
1.23637 & -2.6581 & -1.12503
\end{array}\right] \mathrm{kg}^{1 / 2} \cdot \mathrm{m} / \mathrm{s}^{2},
$$

respectively. The first four elastic modes that have been considered in the model used for simulating a spacecraft are $\omega_{n 1}=0.7681, \omega_{n 2}=1.1038, \omega_{n 3}=1.8733$, and $\omega_{n 4}=$ $2.5496 \mathrm{rad} / \mathrm{sec}$ with damping $\xi_{1}=0.0056, \xi_{2}=0.0086, \xi_{3}=$ 0.013 , and $\xi_{1}=0.025$. The initial states of the rotation motion are given by $Q(0)=\left[\begin{array}{llll}0.3 & -0.1 & 0.2 & 0.9274\end{array}\right]^{T}, \quad \omega(0)=$ $\left[\begin{array}{lll}0 & 0 & 0\end{array}\right]^{T} \mathrm{rad} / \mathrm{sec}$, and $\vartheta(0)=\left[\begin{array}{lllllll}0 & 0 & 0 & 0 & 0 & 0 & 0\end{array}\right]^{T}$. For the FTCLF (29), the parameters $\beta$ and $\gamma$ are set as $\beta=10$ and $\gamma=2.5$. The adapted ESO parameters are selected as $\lambda=0.5$, $\lambda_{1 i}=2.0, \lambda_{2 i}=1.5$, and $\mu_{i}=5.0(i=1,2,3)$. The attitude control problem is considered in the presence of external disturbance $d(t)$. The external disturbances are described as

$$
d(t)=\left[\begin{array}{c}
0.3 \cos (0.1 t)+0.1 \\
0.15 \sin (0.1 t)+0.3 \cos (0.1 t) \\
0.3 \sin (0.1 t)+0.1
\end{array}\right] \mathrm{Nm}
$$

and the desired angular velocity tracking is given by

$$
\omega_{d}(t)=\left[\begin{array}{c}
-0.04 \cos (0.2 t) \\
-0.04 \sin (0.2 t) \\
0.05 \sin (0.2 t)+\cos (0.2 t)
\end{array}\right] \mathrm{rad} / \mathrm{sec}
$$

together with

$$
q_{d}(0)=\left[\begin{array}{lll}
0 & 0 & 0
\end{array}\right]^{T} .
$$

Note that the optimal Lyapunov SM controller (4.3) in [23] was designed for optimal attitude stabilization. To apply this controller to the tracking problem, we define the total disturbance as

$$
\begin{aligned}
\tau_{d}(t)= & -\omega_{e}^{\times} J \omega_{d}-\omega_{d}^{\times} J_{m b} \omega_{e}-\omega_{d}^{\times} J \omega_{d}-\omega_{d}^{\times} \delta^{T} \psi \\
& -J_{m b} \dot{\omega}_{d}+d(t)
\end{aligned}
$$

and use it as the disturbance in the spacecraft model (24). Also, the corresponding parameters in the optimal Lyapunov SM controller (4.3) in [23] are selected. In this paper, to apply controller (23) in [37] to the attitude control problem we use quaternions instead of Euler angles to describe the attitude of 


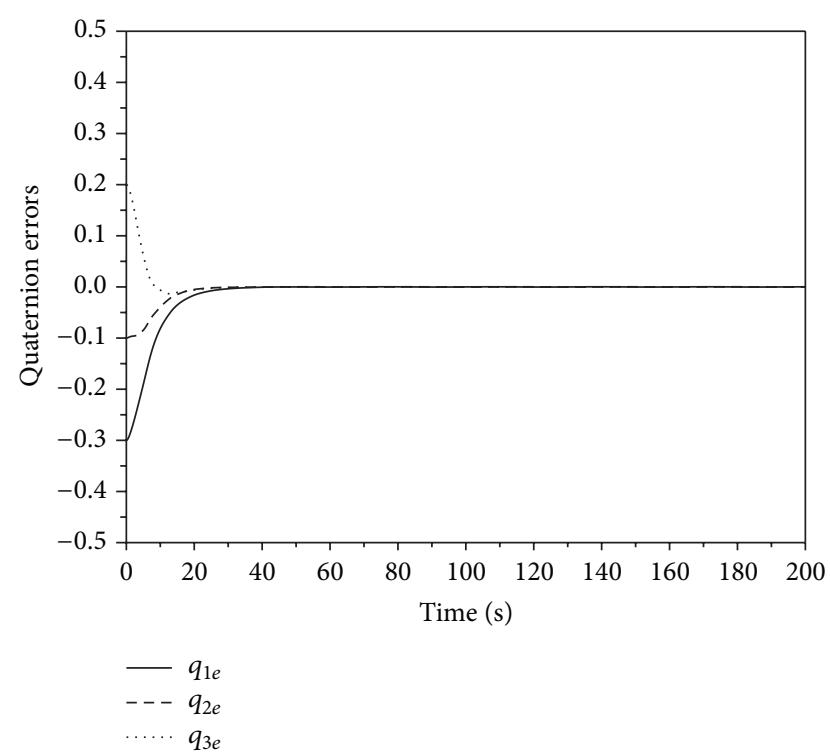

FIGURE 1: Quaternion tracking errors-controller (4.3) in [23].

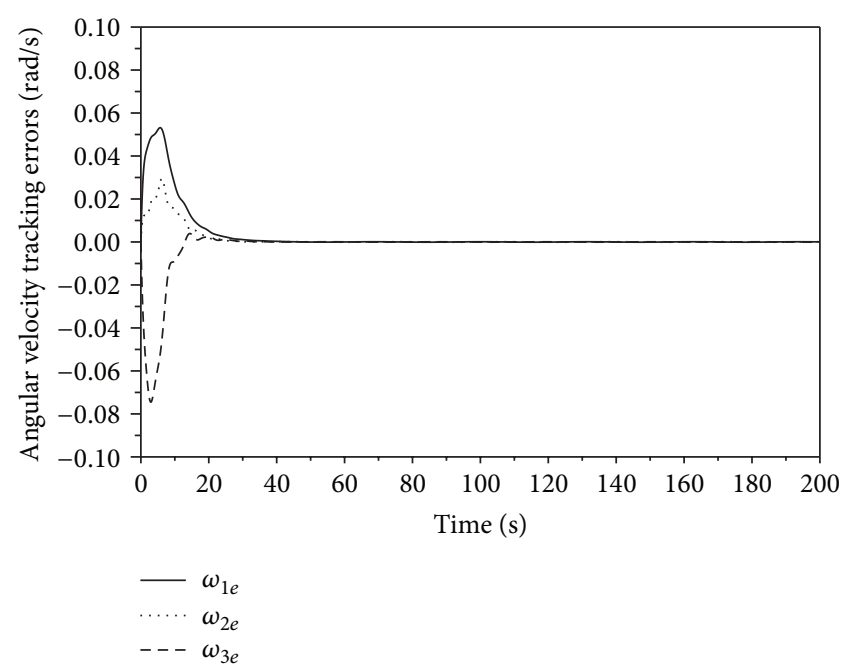

FIGURE 2: Angular velocity tracking errors—controller (4.3) in [23].

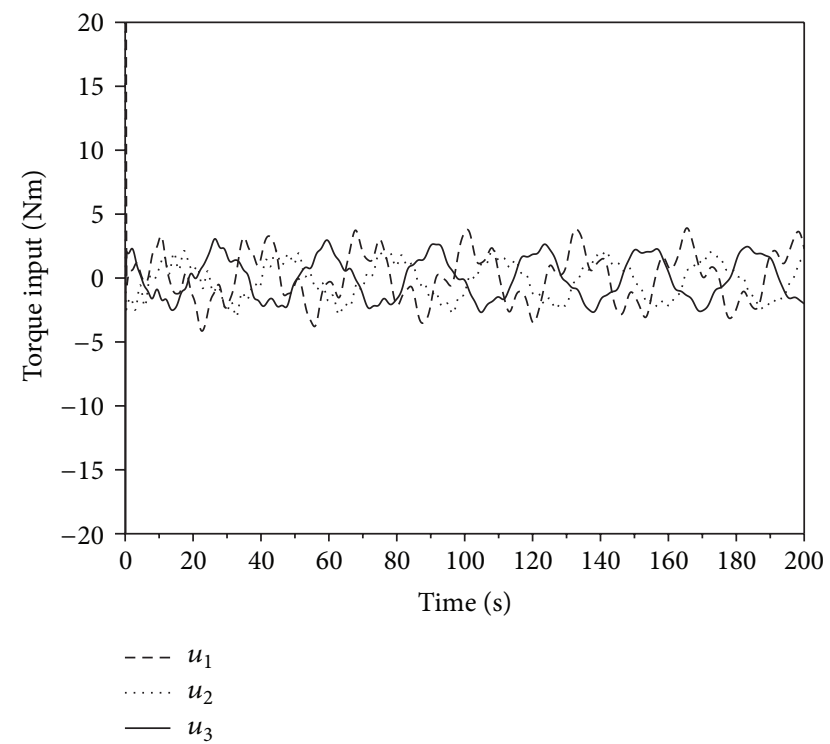

Figure 3: Control torques-controller (4.3) in [23].

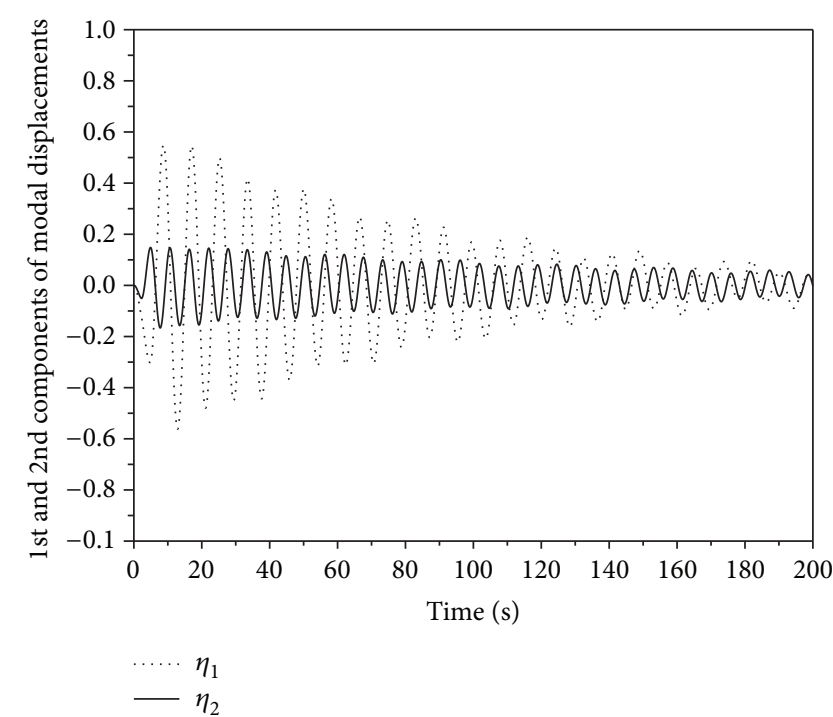

Figure 4: Modal displacements-controller (4.3) in [23].

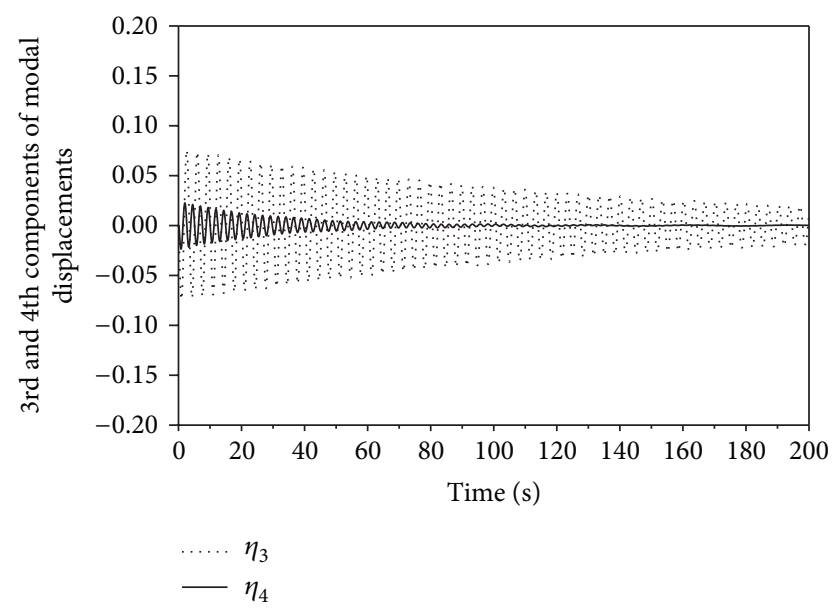

Figure 5: Modal displacements-controller (4.3) in [23].

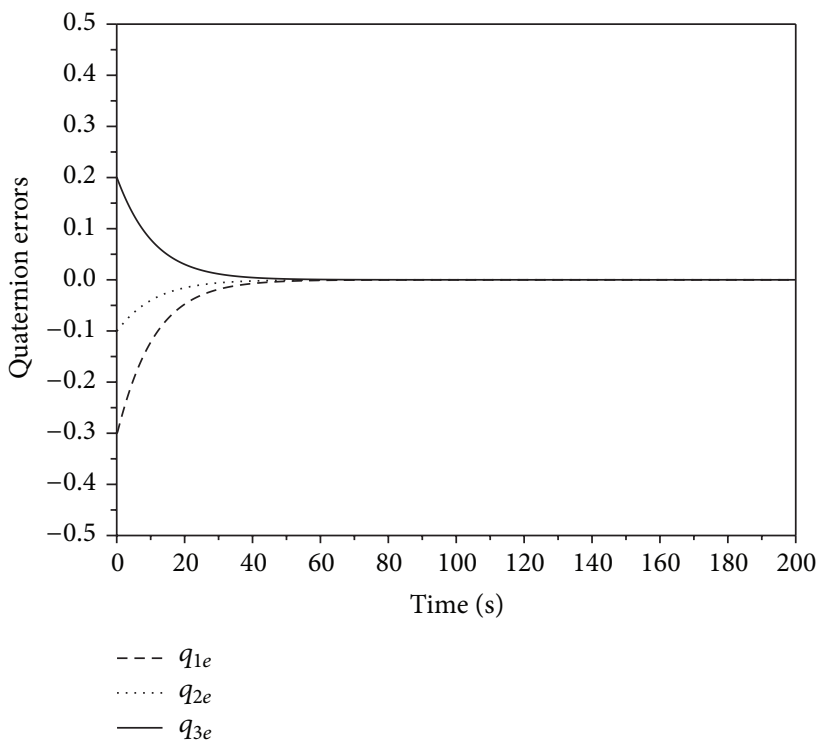

FIgURE 6: Quaternion tracking errors-controller (70). 


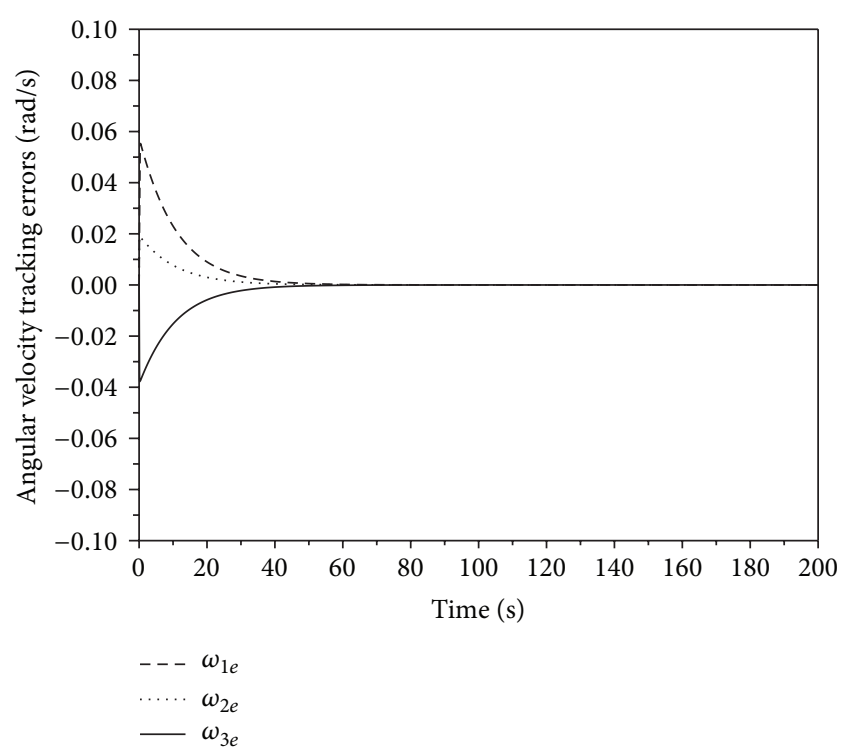

FIGURE 7: Angular velocity tracking errors-controller (70).

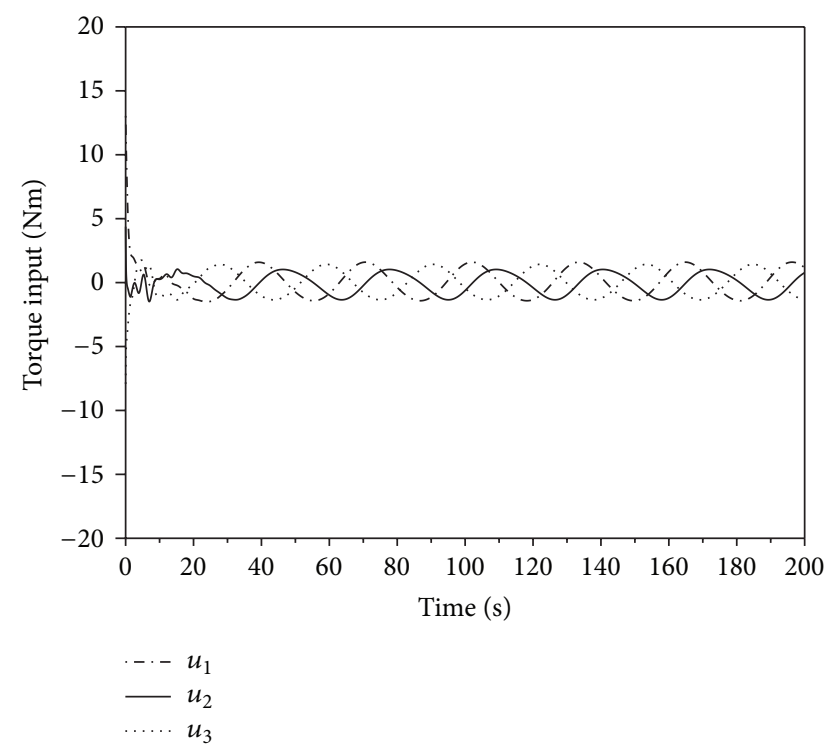

Figure 8: Control torques-controller (70).

the flexible spacecraft. For controller (23) in [37], the control parameters were selected as $\lambda=0.5, \beta=1, a=5, b=7$, and $k^{*}=10 I_{3}$.

Simulation studies have been performed to test all controllers. Figures 1 and 2 show the performance of controller (4.3) in [23]. The responses of quaternion and angular velocity tracking errors reach zero after 40 seconds. The components of angular velocity tracking error vector are smooth. From Figure 3 it can be seen that the optimal Lyapunov SM controller (4.3) in [23] stabilizes the closed-loop system of flexible spacecraft. As shown in Figures 4 and 5 the modal displacements $\left(\eta_{1}-\eta_{4}\right)$ converge to the neighborhood of zero.

On the other hand Figure 6 shows that controller (70) provides good trajectories of the quaternion error and they

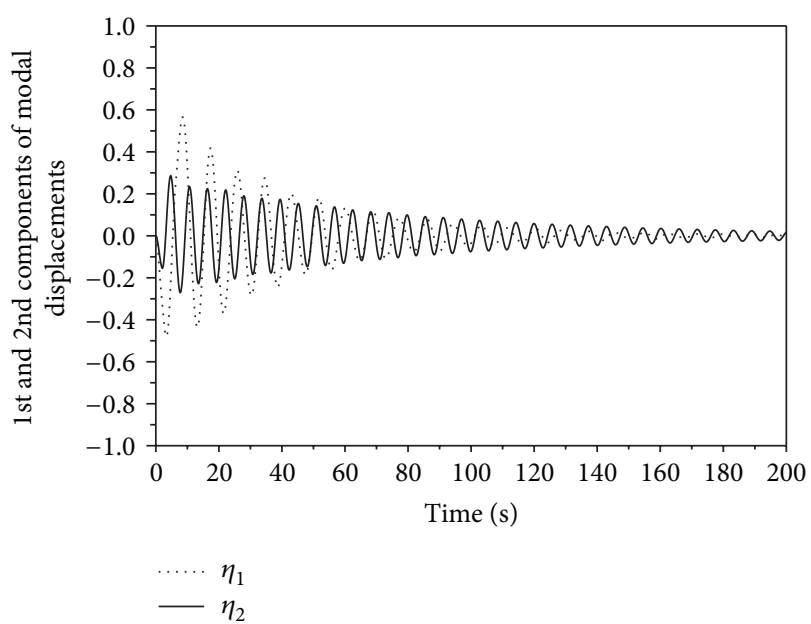

FIGURE 9: Modal displacements—controller (70).

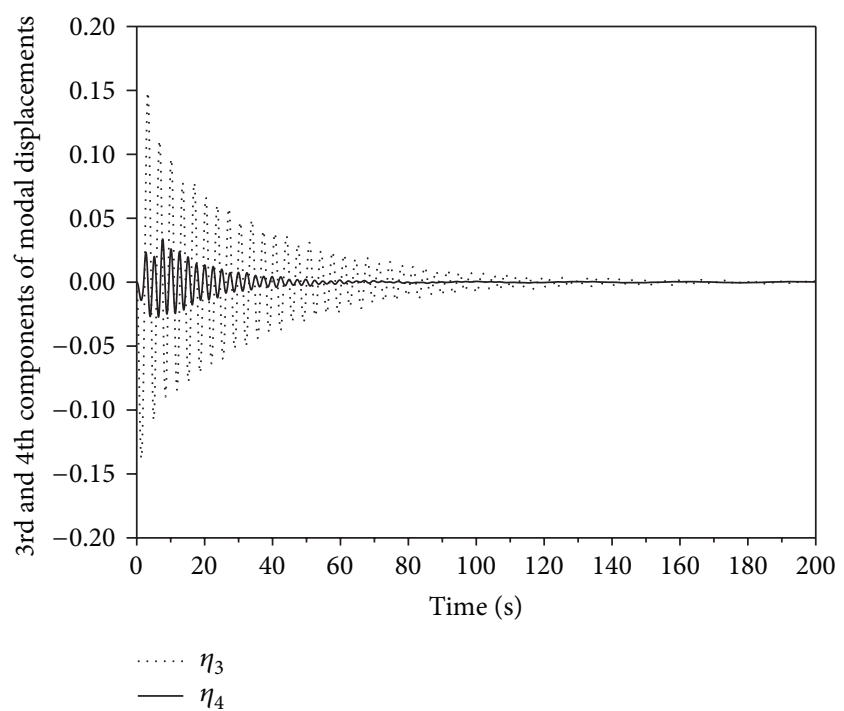

FIGURE 10: Modal displacements-controller (70).

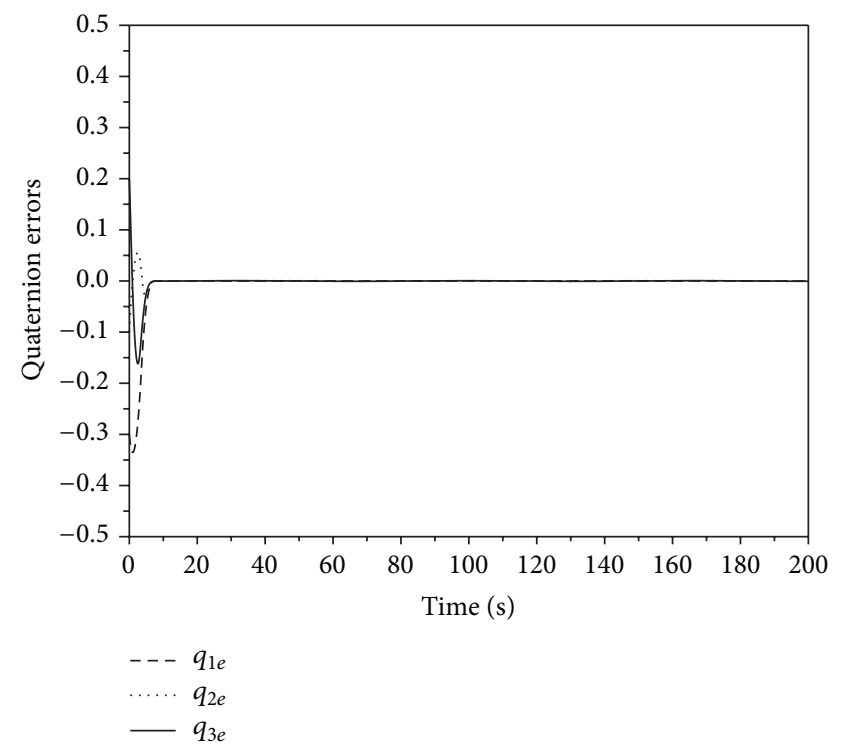

FIGURE 11: Quaternion tracking errors-controller (23) in [37]. 


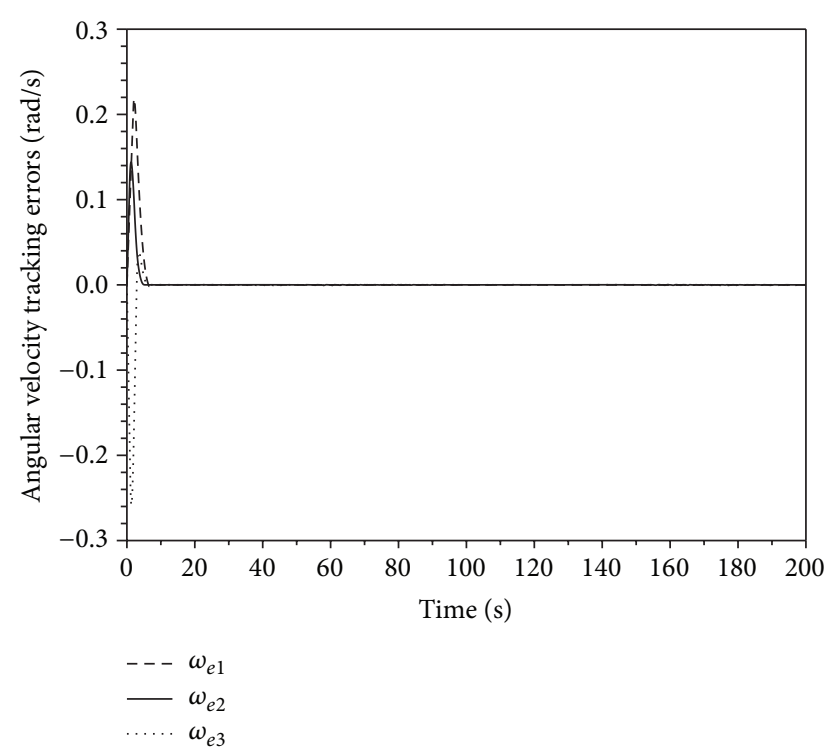

FIGURE 12: Angular velocity tracking errors-controller (23) in [37].

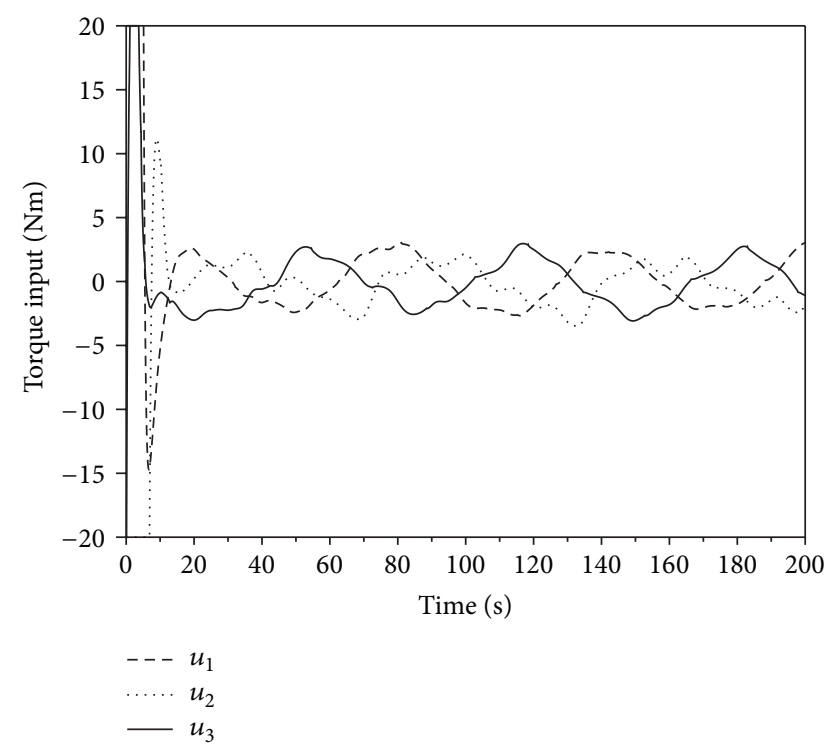

FIGURE 13: Control torques-controller (23) in [37].

reach zero in about 50 seconds. Similarly, from Figure 7 it can be seen that the angular velocity tracking errors reach zero after 80 seconds. The responses of angular velocity tracking errors at the first 20 seconds are smoother when compared with those obtained from controller (4.3) in [23]. As shown in Figure 8 the control torques obtained by controller (70) are quite smooth even though the external disturbances are taken into account. The responses of modal displacements shown in Figures 9 and 10 converge to a smaller region around the zero when compared to controller (4.3) in [23]. Figures 1115 show the simulation results of controller (23) in [37]. As shown in Figures 11 and 12 quaternion and angular velocity tracking errors converge to zero in about 10 seconds. Figure 13 depicts the control torques which approximate the harmonic

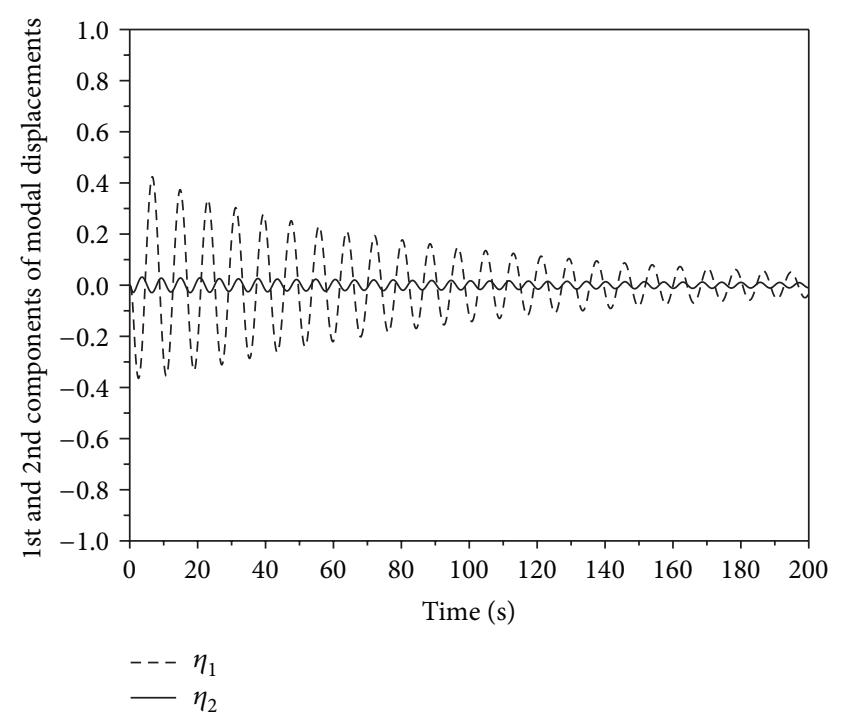

FIGURE 14: Modal displacements-controller (23) in [37].

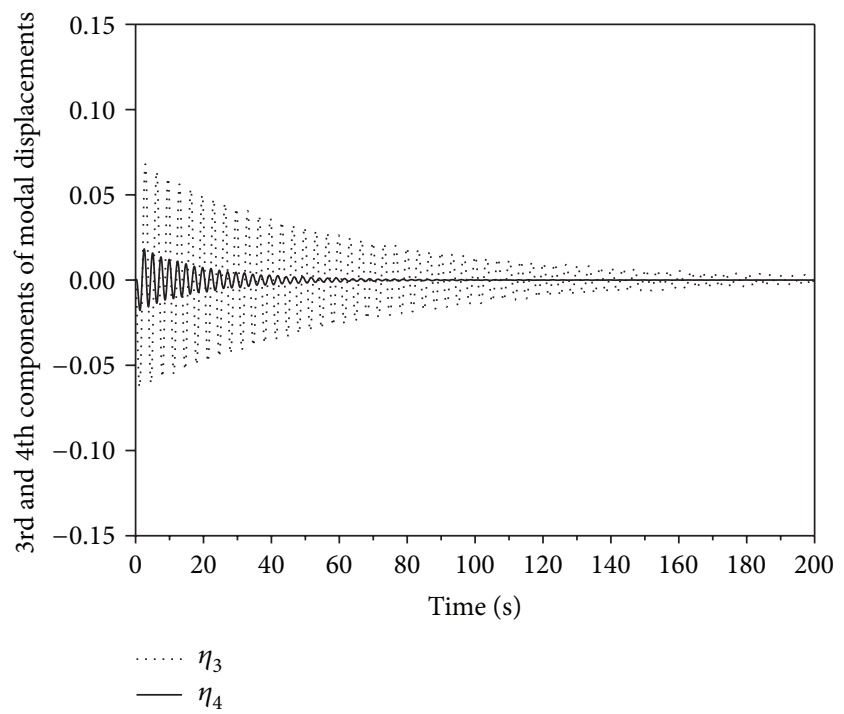

FIGURE 15: Modal displacements-controller (23) in [37].

curves. The responses of modal displacements for controller (23) in [37] are shown in Figures 14 and 15.

A comparison of the simulation results obtained by control law (4.3) in [23], our proposed controller (70), and controller (23) in [37] shows the following. It can be seen that our proposed control law (70) provides smoother attitude velocity tracking error responses and control input responses than those of controller (4.3) in [23]. It can be seen that the vibration of flexible appendages of the spacecraft is obviously reduced by applying the proposed controller (70) rather than through the use of (4.3) in [23]. Next, we compare the performance of (70) and controller (23) in [37]. Although tracking responses obtained by our proposed control law (70) converge more slowly to zero than (23) in [37], smaller values of control torques are required in (70) than in (29). Our proposed control law is based on the inverse optimal control concept that minimizes the performance index, so 
it usually requires smaller values of control torques. In addition, the proposed controller gives smoother control torque signals. This shows that our controller (70) achieves better disturbance rejection performance than (23) in [37]. In view of these simulation results, controller (70) seems to give the best overall control for practical inverse optimal attitude tracking control of a flexible spacecraft.

\section{Conclusion}

We have studied a finite-time anti-disturbance inverse optimal controller design of attitude tracking of a flexible spacecraft in the presence of external disturbances. The concepts of inverse optimal control and the FTCLF have been employed to develop a novel finite-time inverse optimal attitude tracking control law. An adapted ESO has been designed by modifying the structure of the traditional ESO. The finitetime convergence of an adapted ESO has been proven using the strict Lyapunov function. A finite-time anti-disturbance inverse optimal controller can be expressed as the sum of a finite-time inverse optimal control and adapted ESO. It has been shown that the developed controller solves the inverse optimal control problem and converges to the reference attitude states in finite time. An example of multiaxial attitude maneuver is presented and simulation results are given and compared with the results from controller (4.3) in [23] and controller (23) in [37] to verify the usefulness of the developed controller.

\section{References}

[1] S. R. Vadali, L. G. Kraige, and J. L. Junkins, "New results on the optimal spacecraft attitude maneuver problem," Journal of Guidance, Control, and Dynamics, vol. 7, no. 3, pp. 378-380, 1984.

[2] S. Bharadwaj, M. Osipchuk, K. D. Mease, and F. C. Park, "Geometry and inverse optimality in global attitude stabilization," Journal of Guidance, Control, and Dynamics, vol. 21, no. 6, pp. 930939, 1998.

[3] C. K. Carrington and J. L. Junkins, "Optimal nonlinear feedack control for spacecraft attitude maneuvers," Journal of Guidance, Control, and Dynamics, vol. 9, no. 1, pp. 99-107, 1986.

[4] C.-C. Yang and C.-J. Wu, "Optimal large-angle attitude control of rigid spacecraft by momentum transfer," IET Control Theory and Applications, vol. 1, no. 3, pp. 657-664, 2007.

[5] S.-P. Shue, M. E. Sawan, and K. Rokhsaz, "Optimal feedback control of a nonlinear system: wing rock example," Journal of Guidance, Control, and Dynamics, vol. 19, no. 1, pp. 166-171, 1996.

[6] R. Sharma and A. Tewari, "Optimal nonlinear tracking of spacecraft attitude maneuvers," IEEE Transactions on Control Systems Technology, vol. 12, no. 5, pp. 677-682, 2004.

[7] A. El-Gohary, "Optimal control of a programmed motion of a rigid spacecraft using redundant kinematics parameterizations," Chaos, Solitons and Fractals, vol. 26, no. 4, pp. 1053-1063, 2005.

[8] D. K. Parrish and D. B. Ridgely, "Attitude control of a satellite using the SDRE method," in Proceedings of the American Control Conference, pp. 942-946, Albuquerque, NM, USA, June 1997.
[9] D. T. Stansbery and J. R. Cloutier, "Position and attitude control of a spacecraft using the state-dependent Riccati equation technique," in Proceedings of the American Control Conference, pp. 1867-1871, Chicago, Ill, USA, June 2000.

[10] P. Tsiotras, "Stabilization and optimality results for the attitude control problem," Journal of Guidance, Control, and Dynamics, vol. 19, no. 4, pp. 772-779, 1996.

[11] M. Krstić and P. Tsiotras, "Inverse optimal stabilization of a rigid spacecraft," IEEE Transactions on Automatic Control, vol. 44, no. 5, pp. 1042-1049, 1999.

[12] Y. Park, "Inverse optimal and robust nonlinear attitude control of rigid spacecraft," Aerospace Science and Technology, vol. 28, no. 1, pp. 257-265, 2013.

[13] E. D. Sontag, "A "universal" construction of Artstein's theorem on nonlinear stabilization," Systems and Control Letters, vol. 13, no. 2, pp. 117-123, 1989.

[14] R. A. Freeman and P. V. Kokotovic, "Inverse optimality in robust stabilization," SIAM Journal on Control and Optimization, vol. 34, no. 4, pp. 1365-1391, 1996.

[15] W. Kang, "Nonlinear $\mathrm{H}_{\infty}$ control and its application to rigid spacecraft," IEEE Transactions on Automatic Control, vol. 40, no. 7, pp. 1281-1285, 1995.

[16] W. Luo, Y.-C. Chu, and K.-V. Ling, "Inverse optimal adaptive control for attitude tracking of spacecraft," IEEE Transactions on Automatic Control, vol. 50, no. 11, pp. 1639-1654, 2005.

[17] Y. Park, "Robust and optimal attitude stabilization of spacecraft with external disturbances," Aerospace Science and Technology, vol. 9, no. 3, pp. 253-259, 2005.

[18] J. Han, "From PID to active disturbance rejection control," IEEE Transactions on Industrial Electronics, vol. 56, no. 3, pp. $900-$ 906, 2009.

[19] Y. Xia, Z. Zhu, M. Fu, and S. Wang, "Attitude tracking of rigid spacecraft with bounded disturbances," IEEE Transactions on Industrial Electronics, vol. 58, no. 2, pp. 647-659, 2011.

[20] V. I. Utkin, Sliding Modes in Control and Optimization, Springer, Berlin, Germany, 1992.

[21] C. Pukdeboon and A. S. I. Zinober, "Optimal sliding mode controllers for attitude tracking of spacecraft," in Proceedings of the IEEE International Conference on Control Applications, pp. 1708-1713, Saint Petersburg, Russia, July 2009.

[22] C. Pukdeboon and A. S. I. Zinober, "Control Lyapunov function optimal sliding mode controllers for attitude tracking of spacecraft," Journal of the Franklin Institute, vol. 349, no. 2, pp. 456475, 2012.

[23] C. Pukdeboon, "Optimal sliding mode controllers for attitude stabilization of flexible spacecraft," Mathematical Problems in Engineering, vol. 2011, Article ID 863092, 20 pages, 2011.

[24] N. N. Krasovskii, "On the stabilization of unstable motions by additional forces when the feedback loop is incomplete," Journal of Applied Mathematics and Mechanics, vol. 27, no. 4, pp. 9711004, 1963.

[25] E. D. Sontag, "Mathematical control theory," in Deterministic Finite Dimensional Systems, vol. 6 of Texts in Applied Mathematics, Springer, New York, NY, USA, 2nd edition, 1998.

[26] J. A. Primbs, V. Nevistić, and J. C. Doyle, "Nonlinear optimal control : a control Lyapunov function and receding horizon perspective," Asian Journal of Control, vol. 1, no. 1, pp. 14-24, 1999.

[27] X. Cai, "Finite time inverse optimal control of affine nonlinear systems," in Proceedings of the 24th Chinese Control and Decision Conference, Taiyuan, China, May 2012. 
[28] L. Mo, "Finite-time $H_{\infty}$ inverse optimal control of affine nonlinear systems," Circuits, Systems, and Signal Processing, vol. 32, no. 1, pp. 47-60, 2013.

[29] S. Li, X. Yang, and D. Yang, "Active disturbance rejection control for high pointing accuracy and rotation speed," Automatica, vol. 45, no. 8, pp. 1854-1860, 2009.

[30] Y. Huang and J. Han, "Analysis and design for the second order nonlinear continuous extended states observer," Chinese Science Bulletin, vol. 45, no. 21, pp. 1938-1944, 2000.

[31] J. R. Wertz, Spacecraft Attitude Determination and Control, Kluwer Academic, Dordrecht, The Netherlands, 1978.

[32] S. Di Gennaro, "Passive attitude control of flexible spacecraft from quaternion measurements," Journal of Optimization Theory and Applications, vol. 116, no. 1, pp. 41-60, 2003.

[33] V. T. Haimo, "Finite time controllers," SIAM Journal on Control and Optimization, vol. 24, no. 4, pp. 760-770, 1986.

[34] S. P. Bhat and D. S. Bernstein, "Finite-time stability of continuous autonomous systems," SIAM Journal on Control and Optimization, vol. 38, no. 3, pp. 751-766, 2000.

[35] M. D. Shuster, "Survey of attitude representations," Journal of the Astronautical Sciences, vol. 41, no. 4, pp. 439-517, 1993.

[36] S. Di Gennaro, "Output attitude tracking for flexible spacecraft," Automatica, vol. 38, no. 10, pp. 1719-1726, 2002.

[37] S. Wu, G. Radice, and Z. Sun, "Robust finite-time control for flexible spacecraft attitude maneuver," Journal of Aerospace Engineering, 2012.

[38] Q. Hu, "Sliding mode attitude control with L2-gain performance and vibration reduction of flexible spacecraft with actuator dynamics," Acta Astronautica, vol. 67, no. 5-6, pp. 572-583, 2010. 


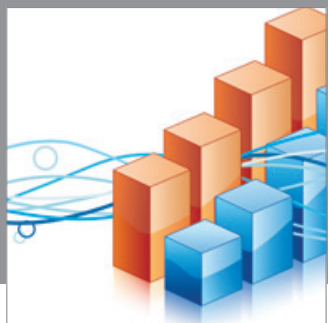

Advances in

Operations Research

mansans

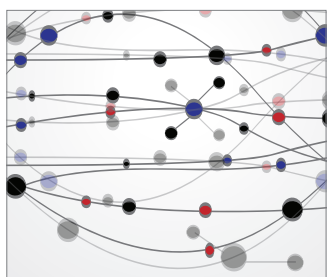

The Scientific World Journal
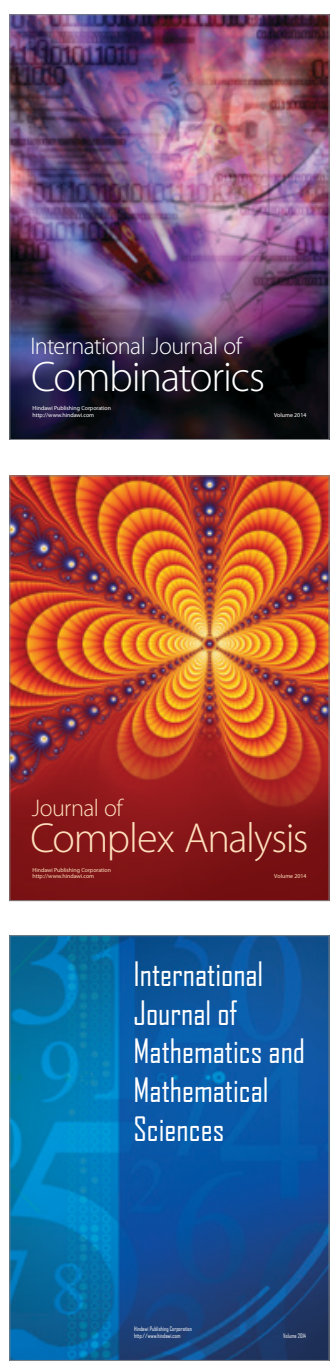
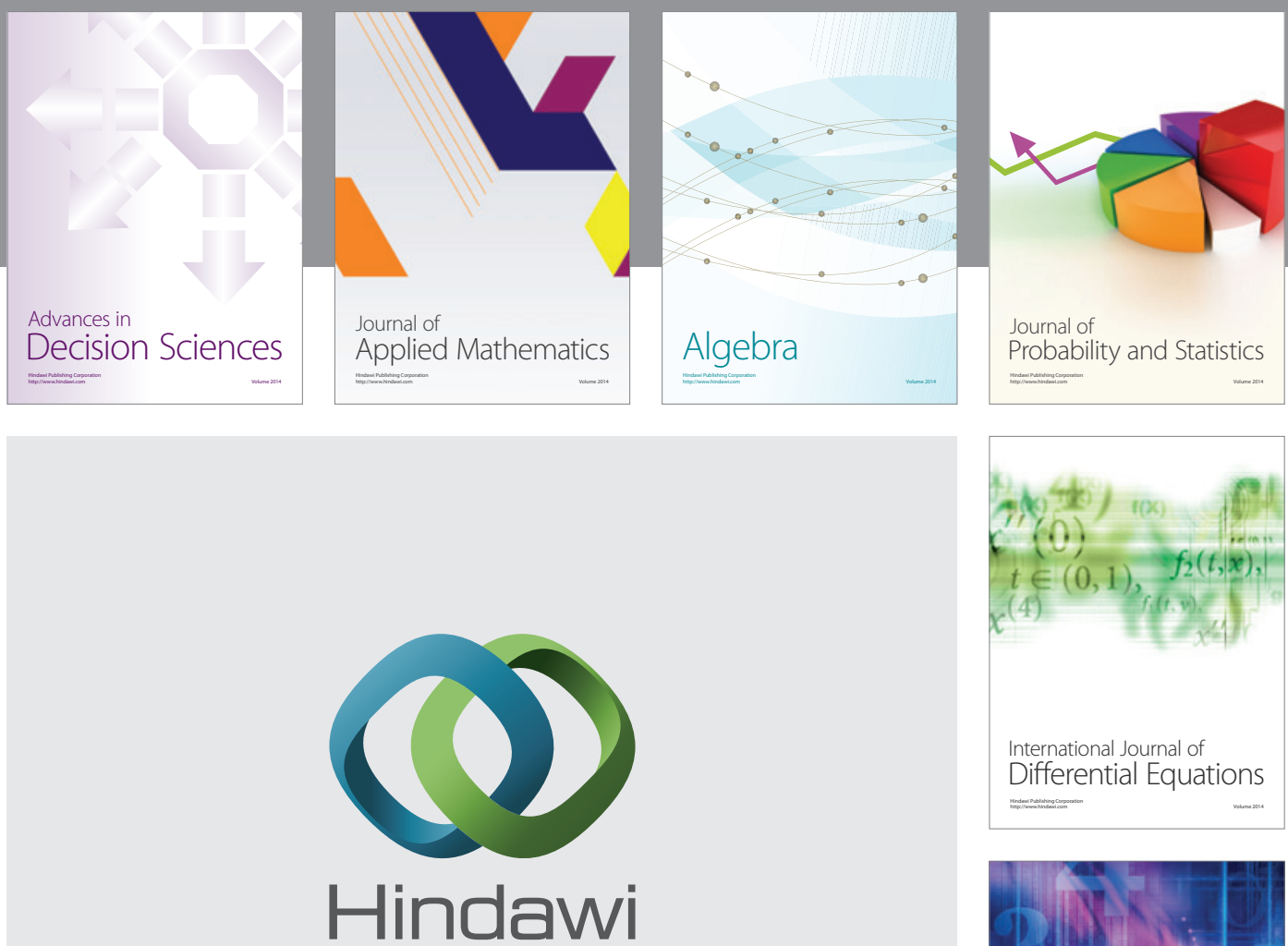

Submit your manuscripts at http://www.hindawi.com
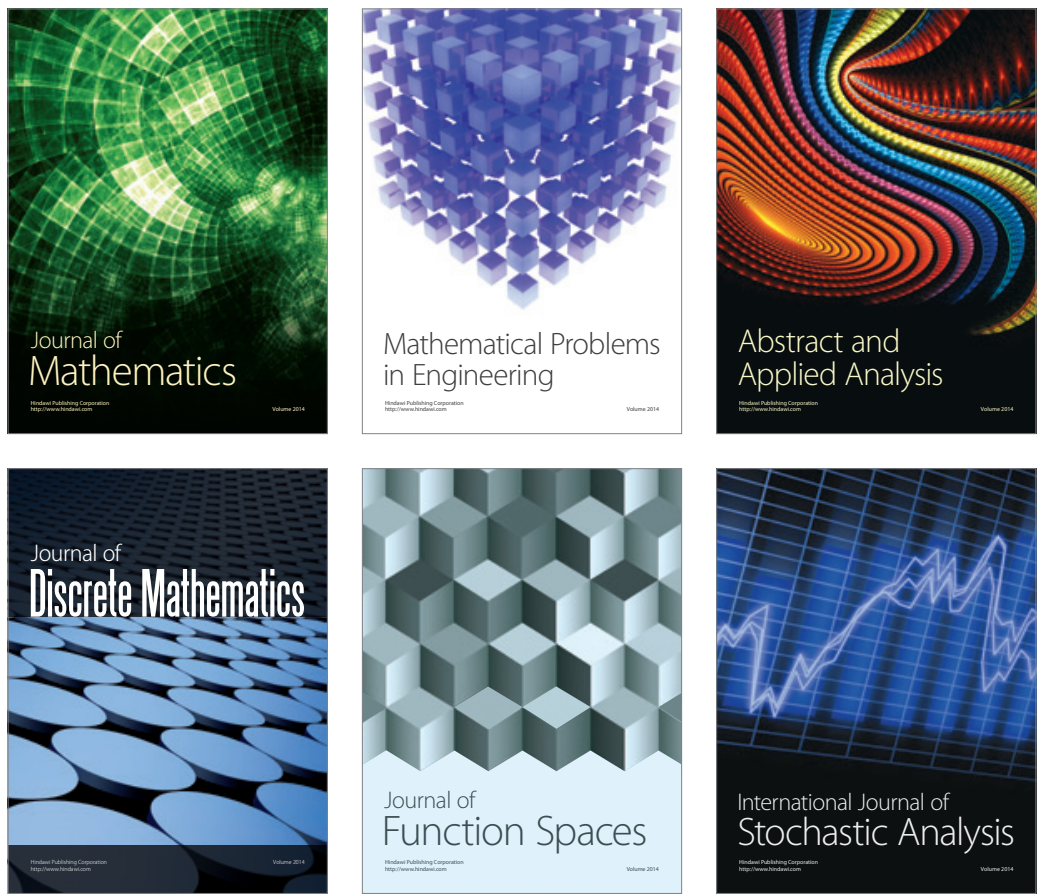

Journal of

Function Spaces

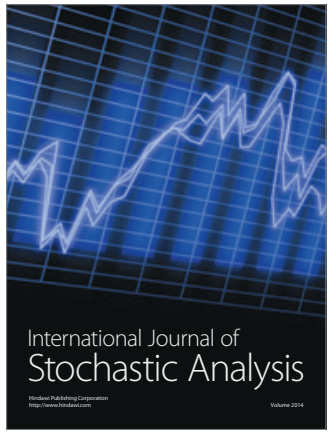

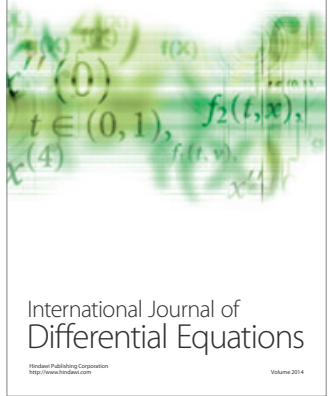
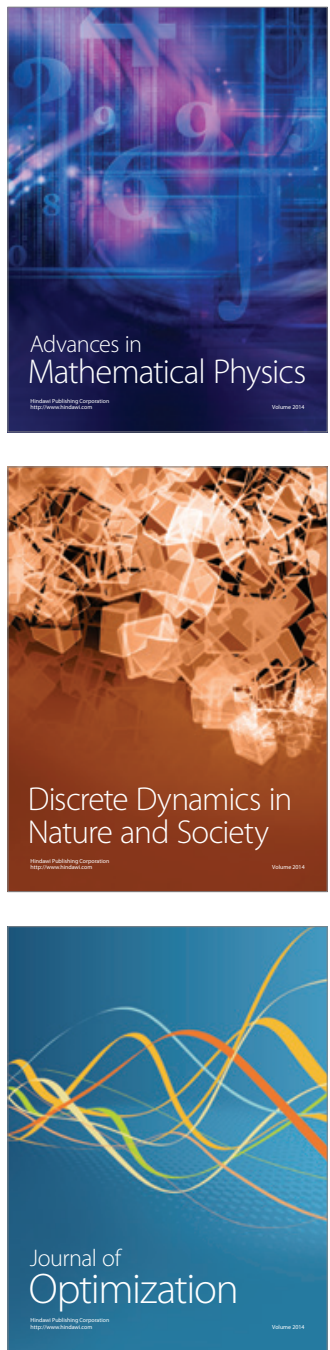